« Migrant » et " réfugié » dans des corpus de presse et des banques de données terminologiques : instabilité des dénominations dans les pratiques langagières

"Migrant » and " réfugié » in press corpora and terminology databases: instability of denominations in language practices

\title{
Claudio Grimaldi
}

\section{(2) OpenEdition}

Journals

\section{Édition électronique}

URL : http://journals.openedition.org/rief/3157

DOI : 10.4000/rief.3157

ISSN : 2240-7456

\section{Éditeur}

Seminario di filologia francese

\section{Référence électronique}

Claudio Grimaldi, « «Migrant » et « réfugié » dans des corpus de presse et des banques de données terminologiques : instabilité des dénominations dans les pratiques langagières », Revue italienne d'études françaises [En ligne], 9 | 2019, mis en ligne le 15 novembre 2019, consulté le 06 août 2020. URL : http://journals.openedition.org/rief/3157 ; DOI : https://doi.org/10.4000/rief.3157

Ce document a été généré automatiquement le 6 août 2020.

\section{cc) (요 $\ominus$}

Les contenus de la RIEF sont mis à disposition selon les termes de la Licence Creative Commons Attribution - Pas d'Utilisation Commerciale - Pas de Modification 4.0 International. 


\section{« Migrant » et « réfugié » dans des corpus de presse et des banques de données terminologiques : instabilité des dénominations dans les pratiques langagières}

"Migrant " and "réfugié " in press corpora and terminology databases: instability of denominations in language practices

Claudio Grimaldi

\section{Introduction}

1 Au cours des dernières années, la crise migratoire est devenue un des sujets politiques, économiques et sociaux parmi les plus débattus de l'Union européenne. Le nombre des flux migratoires a, en effet, atteint une proportion qui ne pouvait pas être prévisible et le drame quotidien des migrants est depuis plusieurs mois à la une de la presse européenne. Face à cette catastrophe humaine d'ampleur inédite, les instances politiques nationales essaient de proposer des solutions afin de catégoriser les immigrés, au moyen d'une politique migratoire dont les actions se basent souvent sur la dichotomie ontologique existant entre « migrant » et « réfugié ».

Parallèlement aux interrogations relatives aux modalités d'accueil des personnes traversant la Méditerranée ou rejoignant l'Europe par d'autres voies, un débat sémantique semble s'instaurer autour des catégories classifiant les personnes qui fuient leurs pays pour de multiples raisons. C'est au sein de ce débat que s'inscrit notre réflexion concernant la terminologie relative aux flux migratoires.

Dans notre étude il s'agira, d'une part, de décrire la problématique liée aux concepts de « migrant »/« réfugié », vu le foisonnement de plusieurs dénominations intermédiaires 
nouvelles (par exemple «migrant/réfugié économique», «migrant/réfugié climatique ", "écoréfugié ", "migrant/réfugié environnemental »), utilisées pour catégoriser les personnes fuyant leur propre pays, ainsi que, d'un point de vue principalement lexico-sémantique ${ }^{1}$, de réfléchir sur le débat existant sur le plan de l'usage en discours des mots « migrant » et " réfugié ». D'autre part, nous présenterons les dénominations nouvelles apparues au cours des dernières années et utilisées pour créer des catégories de migrants et réfugiés qui peuvent être exploitées sur le plan juridique, faute de dénominations partagées au niveau normatif. Pour cette dernière partie de notre contribution, nous nous insérons dans le cadre des réflexions proposées par Sablayrolles ${ }^{2}$ sur le concept de néologie combinatoire.

4 Notre étude, qui porte exclusivement sur le vocabulaire français de la migration, bien qu'elle s'insère dans un cadre de réflexion linguistique international ${ }^{3}$, s'appuiera sur l'exploitation de deux typologies de corpus complémentaires afin de comprendre les différents aspects sociaux, politiques et juridiques impliqués dans la terminologie des flux migratoires. Le premier corpus (Corpus 1), que nous pouvons qualifier de textuel, est constitué des discours médiatiques concernant la question migratoire. Les données retenues, tirées de l'enquête réalisée en 2015 par la Coordination 75 des Sans Papiers de Paris et de la plateforme Néoveille ${ }^{4}$, permettront d'encadrer les problèmes liés à l'usage en discours des mots "migrant » et "réfugié " dans la sphère du débat public. Le second corpus (Corpus 2), que nous pouvons qualifier de secondaire, puisqu'il est constitué des extraits de banques de données terminologiques en ligne telles que le Grand Dictionnaire Terminologique ${ }^{5}$ (GDT), FranceTerme ${ }^{6}$ (FT) et Termium $^{7}$ (T), permettra de recenser les dénominations nouvelles créées pour catégoriser les concepts de migrant et réfugié. Finalement, dans 2.2, nous proposerons une réflexion sur les procédés d'arrangement sémantique des composantes des syntagmes retenus.

\section{1. "Migrant " vs « réfugié »: une dichotomie conceptuelle désormais inadéquate}

5 La problématique liée à la catégorisation des personnes fuyant leur propre pays est née principalement au sein des discours médiatiques, surtout en 2015 lorsque le journaliste Barry Malone commenta la décision prise par la chaîne Al Jazeera de bannir le terme correspondant au concept de "migrant» de son antenne et de lui préférer celui de "réfugié »8. Selon le journaliste, "migrant», qui renvoie à "immigrant», terme connoté d'ailleurs très négativement en France, est un mot qui ôte la parole à des personnes en souffrance et le fait de lui substituer le terme « réfugié » est au moins une tentative de leur en redonner un peu. Cette réflexion a rapidement enchaîné une série d'autres interrogations au niveau supranational concernant la manière d'appeler la crise migratoire. Toujours en 2015, Bertrand Vannier, médiateur de Radio France ${ }^{9}$, a en effet indiqué que le débat autour des termes à utiliser dans ce contexte est un sujet cher à plusieurs auditeurs qui reprochent aux représentants politiques et aux journalistes d'oublier le poids des mots, en soulignant que des difficultés majeures résident dans le constat que la géopolitique et la politique se mêlent et parfois contredisent le droit.

6 Le point discuté à plusieurs reprises concerne la création d'une dichotomie entre les migrants et les réfugiés, des dénominations qui sur le plan linguistique sont souvent utilisées de manière interchangeable en tant que synonymes, alors que des points de différence existent, du moins sur le plan conceptuel. « Migrant » n'indique pas, en effet, 
une catégorie juridique ${ }^{10}$; ce terme désigne simplement les personnes qui, par choix, par nécessité ou par contrainte, quittent leur pays pour aller s'installer ailleurs. Au contraire, les « réfugiés » jouissent d'un statut encadré par la Convention de Genève de 1951, qui précise également les conditions de l'état juridique de réfugié et les obligations légales des États qui l'ont ratifiée. De ce point de vue, la Convention ne définit pas seulement le cas de l'asile politique, son champ d'application s'avérant bien plus large puisque le statut de réfugié s'applique à toute personne qui, craignant à raison d'être persécutée du fait de sa race, de sa religion, de sa nationalité, de son orientation sexuelle, de son appartenance à un certain groupe social ou de ses opinions politiques, se trouve hors du pays dont elle a la nationalité. Selon la Convention de Genève, le réfugié est aussi une personne n'ayant pas de nationalité, qui se trouve hors de son pays de résidence, dans lequel elle ne peut pas ou ne veut pas y retourner en raison de la crainte d'être persécutée.

7 Bien que cette catégorisation puisse apparaître pragmatiquement très claire et simple à opérer, les études menées en sociologie ${ }^{11}$ préconisent de ne pas effectuer de distinction aussi tranchée. Comme l'indique Akoka ${ }^{12}$, les conceptions de " migrant » et "réfugié » ont sensiblement changé au fil du temps, ce qui a provoqué le passage "d'une conception du "réfugié" marquée par une grande porosité avec la catégorie de "migrant", à la rigidification de la catégorie de "réfugié", puis à sa dualisation avec l'apparition de la catégorie de "demandeur d'asile". Ces transformations doivent être restituées dans le contexte plus large de la crise économique, mais aussi dans celui de la fin de la guerre froide et de la crise de l'État providence». Dans une perspective toujours historique, Akoka a souligné qu'à la variabilité et à l'évolution des termes "migrant» et "réfugié ", dont les dictionnaires français contemporains ont saisi l'élargissement sémantique ${ }^{13}$, s'ajoute aussi une variabilité des interprétations et des catégories. Parallèlement, en l'absence de différences nettes entre réfugiés et migrants en termes de traitement institutionnel et de droits, c'est également le choix subjectif des exilés de se tourner vers l'une ou l'autre procédure (asile ou immigration) qui contribue à leur qualification. Bien loin d'une définition universelle, la qualification de " réfugié » apparaissait ainsi comme le résultat d'un processus à l'intersection entre, d'un côté, des choix subjectifs et, de l'autre, des dispositifs d'action publique participant à l'orientation stratégique des étrangers selon leurs groupes d'appartenance, les besoins sociaux et les intérêts politiques d'une période historique donnée.

8 Si la question de la signification des mots « migrant » et « réfugié » est aussi complexe, c'est aussi parce que des enjeux politiques sont à la base de l'usage de ces dénominations : premièrement, la manière dont on nomme une personne qui ne se sent nécessairement ni réfugié ni migrant implique le droit politique à l'assistance de l'État, à la protection internationale et à des procédures de surveillance spécifique; deuxièmement, les catégories de "migrant » et "réfugié » sont mises en opposition dans le but de créer une hiérarchie morale et politique. On reste donc prisonniers d'une dichotomie qui voudrait que l'on considère deux cas de figure : d'une part, les gens qui se déplacent pour des raisons politiques et peuvent donc prétendre au statut de réfugié et à une protection internationale; de l'autre, les migrants qui se déplacent pour des raisons économiques et que l'on pourrait considérer presque comme des marchandises à renvoyer à l'expéditeur. Comme l'indique Rodier ${ }^{14}$, ce sont des raisons de politique migratoire qui sont à la base de la connotation négative du mot "migrant» (sousentendu « économique »), " comme s'il était en soi condamnable de chercher dans un 
autre pays que le sien des opportunités de vie meilleure - voire de seule subsistance et de survie ».

$9 \quad$ Il est ainsi possible de supposer des périodes d'emploi préférentiel des dénominations dans le débat public où, selon Setti ${ }^{15}$, le mot «migrant» a été chargé d'acceptions généralement associées au concept de "réfugié ", ce qui a affaibli la signification propre au mot "migrant $»^{16}$ et a rendu nécessaire la création de sous-catégories fonctionnelles pour rendre compte des variétés des situations personnelles existant dans l'univers de la migration.

Or, comme nous avons pu le constater, les discours politiques et médiatiques internationaux s'orientent autour de la dichotomie proposée entre "migrant " et « réfugié ». C'est donc sur ce premier volet que nous nous pencherons par la suite afin de vérifier l'emploi dans le débat public de ces deux dénominations françaises et en saisir l'interchangeabilité au niveau discursif.

\section{Les pratiques langagières de dénomination des migrants et réfugiés}

\subsection{Corpus 1 : l'enquête de la CSP75 et la plateforme Néoveille}

11 Une première enquête consacrée à l'emploi dans les discours politiques et médiatiques des désignations des personnes, qui sont arrivées en France lors des nombreux flux migratoires des dernières années, a été réalisée par la Coordination 75 des Sans Papiers de Paris ${ }^{17}$. L'étude, parue en 2015, a été mise à jour en juin 2016 à partir de quelques 1200 références, communiqués, appels, pages d'actualité, lettres d'information, articles de presse ${ }^{18} d$ 'acteurs de la scène sociale française. Cela dans le but de déterminer les occurrences qualifiant les personnes et les populations, et non les formes verbales, ni les expressions telles que, par exemple, "passage clandestin » ou d'autres syntagmes comme « Haut-Commissariat pour les réfugiés ». Les résultats de l'enquête, qui ont été traduits en dessins en reproduisant les pourcentages obtenus dans des topologies des positionnements des mots et de leur emploi, témoignent des choix faits au niveau communicationnel par les acteurs pris en considération. Il s'agit d'institutions, partis politiques, syndicats, organisations, associations et collectifs.

Selon l'enquête menée par la Coordination, une distinction évidente émerge entre un langage pauvre se limitant à l'emploi de "migrant » ou "réfugié » et un langage plus riche qui s'élargit à une pluralité d'expressions, et qui relativise de manière nette l'alternative entre les deux mots. Ce sont notamment les associations et les collectifs de sans papiers, qui sont bien évidemment les plus sensibles à l'emploi d'une terminologie correcte, qui se distinguent par un moindre emploi de "migrant » et " réfugié » au profit d'une variété d'expressions, telles que "demandeur d'asile ", "étranger ", " exilé", "sans papiers", rarement prises en compte par les acteurs politiques. L'objectif de ce choix nous semble celui de vouloir transmettre de façon évidente la diversité des situations personnelles et des raisons sociales poussant à la migration vers la France. En revanche, des termes tels que «clandestin » et «débouté » sont plutôt utilisés par les partis de droite et d'extrême droite, comme le Front National, Debout la France, Les Républicains, Parti de la France, afin de véhiculer une image hostile des réfugiés. 
13 Nous présentons dans les tableaux ci-dessous les données chiffrées relatives à l'emploi des mots retenus pour l'enquête.

Qui dit quoi ?

S'agissant des migrants, réfugiés, demandeurs d'asile et autres exilés...

\begin{tabular}{|c|c|c|c|c|c|c|c|c|c|c|}
\hline \multicolumn{2}{|c|}{ EN CHIFFRES } & 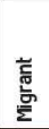 & 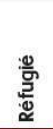 & 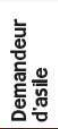 & $\frac{\frac{\omega}{x}}{\mathrm{x}}$ & 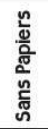 & 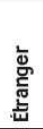 & 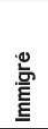 & 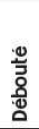 & $\begin{array}{l}\text { 든 } \\
\frac{0}{0} \\
\frac{0}{0} \\
\text { 엉 }\end{array}$ \\
\hline \multirow{9}{*}{$\begin{array}{l}\frac{n}{\vdots} \\
\frac{0}{5} \\
\frac{5}{\underline{n}} \\
\underline{\underline{n}}\end{array}$} & UNHCR - Haut Commissariat des Nations unies pour les réfugiés & $11 \%$ & $76 \%$ & $13 \%$ & $0 \%$ & & & & & $0 \%$ \\
\hline & IOM - Organisation internationale pour les migrations & $75 \%$ & $19 \%$ & $5 \%$ & & $0 \%$ & $0 \%$ & & & $0 \%$ \\
\hline & Parlement européen - UE & $16 \%$ & $46 \%$ & $37 \%$ & & & & & & $1 \%$ \\
\hline & Commission européenne - UE & $33 \%$ & $48 \%$ & $19 \%$ & & & & & & \\
\hline & Conseil européen - UE & $59 \%$ & $29 \%$ & $9 \%$ & & & $1 \%$ & & & $2 \%$ \\
\hline & Gouvernement $-1^{\text {er }}$ ministre & $45 \%$ & $34 \%$ & $17 \%$ & & & $3 \%$ & & $1 \%$ & \\
\hline & Ministère de l'Intérieur & $62 \%$ & $11 \%$ & $21 \%$ & & & $5 \%$ & & $1 \%$ & $0 \%$ \\
\hline & Mairie de Paris > Communiqués de presse & $51 \%$ & $41 \%$ & $7 \%$ & & $1 \%$ & & & & \\
\hline & Mairie de Paris > Site & $26 \%$ & $70 \%$ & $4 \%$ & & & & & & \\
\hline \multirow{12}{*}{$\begin{array}{l}\text { 㟧 } \\
\text { 을 } \\
\text { 옹 }\end{array}$} & FN - Front national & $29 \%$ & $5 \%$ & $17 \%$ & $1 \%$ & & $5 \%$ & $4 \%$ & $9 \%$ & $31 \%$ \\
\hline & PDF - Parti de la France (C. Lang) & $43 \%$ & $24 \%$ & $8 \%$ & & & $3 \%$ & $6 \%$ & $1 \%$ & $16 \%$ \\
\hline & DLF - Debout la France (N. Dupont-Aignan) & $54 \%$ & $5 \%$ & $3 \%$ & & & $17 \%$ & $10 \%$ & $4 \%$ & $8 \%$ \\
\hline & LR - Les Républicains & $42 \%$ & $26 \%$ & $1 \%$ & & $1 \%$ & $19 \%$ & $1 \%$ & $4 \%$ & $6 \%$ \\
\hline & UDI et composantes - Union des démocrates et indépendants & $20 \%$ & $60 \%$ & $20 \%$ & & & & & & \\
\hline & PS - Parti socialiste & $20 \%$ & $54 \%$ & $18 \%$ & & & $2 \%$ & $5 \%$ & & $2 \%$ \\
\hline & EELV - Europe écologie Les Verts & $17 \%$ & $62 \%$ & $8 \%$ & $7 \%$ & & $4 \%$ & $1 \%$ & $1 \%$ & $1 \%$ \\
\hline & PCF Front de gauche - Parti communiste français & $22 \%$ & $67 \%$ & $8 \%$ & & $1 \%$ & & $1 \%$ & & $1 \%$ \\
\hline & PG Front de gauche - Parti de gauche & $39 \%$ & $55 \%$ & $5 \%$ & & $1 \%$ & $1 \%$ & & & $1 \%$ \\
\hline & Ensemble Front de gauche & $42 \%$ & $37 \%$ & $11 \%$ & $1 \%$ & $2 \%$ & $2 \%$ & $5 \%$ & & $1 \%$ \\
\hline & LO - Lutte ouvrière & $45 \%$ & $26 \%$ & $16 \%$ & $1 \%$ & $4 \%$ & $3 \%$ & $3 \%$ & & $1 \%$ \\
\hline & NPA - Nouveau parti anticapitaliste & $56 \%$ & $20 \%$ & $9 \%$ & $0 \%$ & $9 \%$ & $3 \%$ & $1 \%$ & $1 \%$ & $0 \%$ \\
\hline
\end{tabular}

Figure 1. Données chiffrées relatives à l'utilisation des mots analysés dans l'enquête de la CSP75. Les données se réfèrent aux institutions et partis politiques, mars 2018.

En ce qui concerne la presse, nous remarquons que le terme "migrant " occupe une place de choix. Selon les auteurs de l'enquête, les cinq grands quotidiens nationaux testés parlent d'une même voix. Ils emploient les mêmes termes dans des proportions similaires et cela n'est pas dû à la reprise des mêmes dépêches d'agence. Nous exposons ci-après les pourcentages de l'emploi des termes selon l'enquête de la Coordination 75.

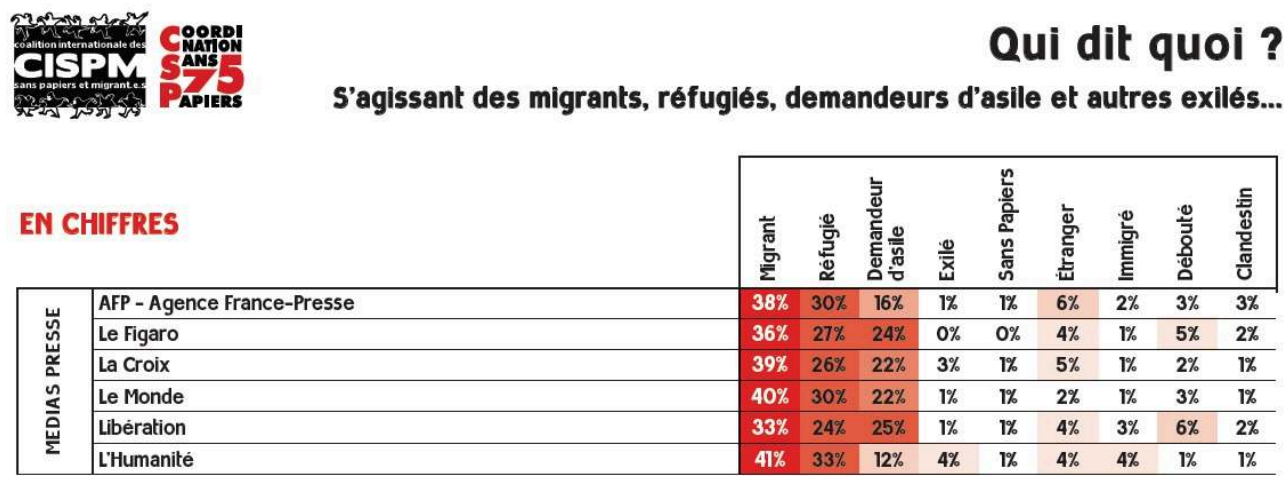

Figure 2. Pourcentages relatifs à l'utilisation des mots analysés dans l'enquête de la CSP75. Les résultats se réfèrent à l'emploi fait par les médias de presse, mars 2018.

15 Afin de vérifier si l'utilisation des termes «migrant » et « réfugié » dans la presse a changé entre 2016 et 2018, nous avons interrogé la plateforme Néoveille, qui permet la consultation en diachronie d'un corpus assez vaste de textes tirés de quotidiens et d'hebdomadaires français, nationaux et régionaux, en proposant également des 
données relatives à l'aire francophone (Belgique, Suisse, pays du Maghreb). Des graphiques et des indications chiffrées peuvent être consultés, ce que nous présentons par la suite en termes de fréquence d'emploi des mots sur la période avril 2017-mars 2018 dans les quotidiens et les hebdomadaires du corpus.

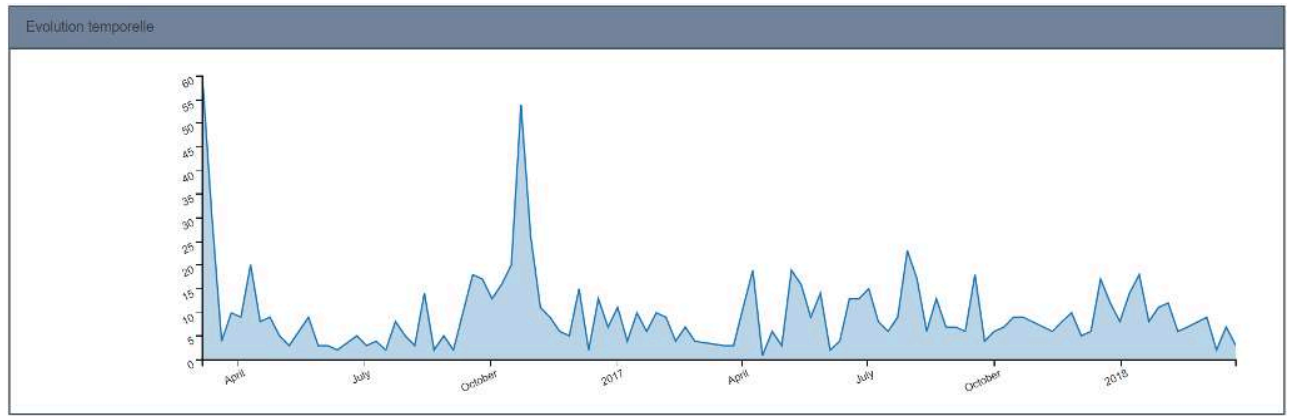

Figure 3. Fréquence d'emploi du mot « migrant » dans le corpus Néoveille, mars 2018.

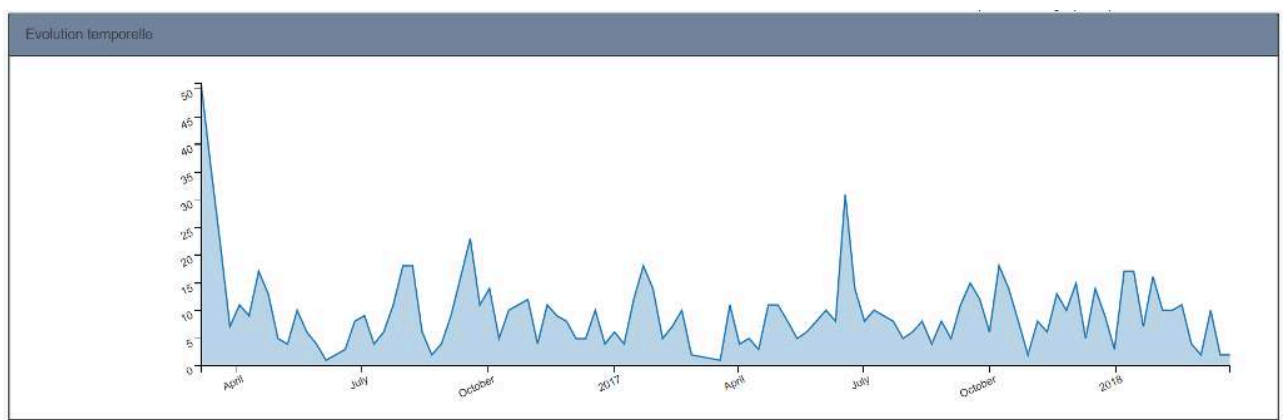

Figure 4. Fréquence d'emploi du mot « réfugié » dans le corpus Néoveille, mars 2018.

La recherche effectuée sur la plateforme Néoveille permet de valider l'hypothèse selon laquelle «migrant » serait utilisé plus fréquemment (15 216 occurrences relevées) que « réfugié ». Quant à l'emploi dans la presse, à l'exception du quotidien La Voix du Nord, la plupart des autres journaux analysés ont recours de manière plus ou moins égale aux deux dénominations. L'emploi quasi exclusif de "migrant» dans La Voix du Nord s'explique par le fait que la question de la «jungle de Calais » et son évacuation ont été à la une de la presse durant plusieurs mois et que le quotidien régional La Voix du Nord a été très probablement celui qui a traité le plus souvent le sujet de la crise migratoire. En ce qui concerne l'emploi dans les grands quotidiens français, nous constatons que Le Monde et Libération emploient ces termes de manière différente, le premier préférant le mot « réfugié ", le second, « migrant ». Enfin, nous constatons que le journal à la ligne éditoriale neutre Le Parisien emploie ces deux mots avec la même fréquence alors que le quotidien La Croix, dont la ligne éditoriale est ouvertement liée à une identité catholique, privilégie le mot "réfugié », pour souligner le besoin d'intégration et de protection des personnes concernées.

\begin{tabular}{|l|l|}
\hline « migrant » & « réfugié » \\
\hline
\end{tabular}




\begin{tabular}{|c|c|}
\hline La Voix du Nord : 208 & L'Express : 86 \\
\hline L'Express : 73 & La Croix : 85 \\
\hline La Nouvelle République: 65 & France Soir : 84 \\
\hline France Soir : 63 & La Nouvelle République: 63 \\
\hline Le Parisien : 41 & Le Parisien : 44 \\
\hline Bien Public : 36 & Le Monde : 35 \\
\hline Le Dauphiné Libéré : 36 & BFM TV : 30 \\
\hline$B F M T V: 35$ & La Libre Belgique : 27 \\
\hline Libération : 35 & Le Dauphiné Libéré : 26 \\
\hline Nice Matin : 31 & La Dépêche du Midi : 26 \\
\hline
\end{tabular}

Table 1. Nombre d'occurrences de « migrant » et « réfugié » dans les médias de presse du corpus Néoveille, mars 2018.

\subsection{Corpus 2 : les banques de données terminologiques en ligne : le Grand Dictionnaire Terminologique, FranceTerme et Termium}

17 Les tentatives de classification des instances politiques analysées auparavant reprennent en partie les distinctions faites au sein du Haut-Commissariat des Nations Unies pour les Réfugiés (UNHCR), qui appelle à ne pas confondre les migrants et les réfugiés afin de proposer à ces derniers toute la protection à laquelle ils ont droit. Toutefois, cette distinction ne tient pas compte du phénomène de la migration dans son ensemble. De ce point de vue, le recours à de nombreux adjectifs accompagnant les termes «migrant »/ » réfugié ", que nous analyserons par la suite, indique l'existence de catégories intermédiaires. De même, certains syntagmes soulignent l'importance des facteurs qui entrent en jeu dans les phénomènes migratoires puisque, comme l'indique Gemenne, " parler de migrants ou de réfugiés environnementaux est aussi une façon de souligner l'importance des facteurs environnementaux dans la décision de migrer plus que la volonté de créer une catégorie spécifique de migration ${ }^{19}$. L'emploi, entre autres, du syntagme "migrant économique " semble renvoyer à cette stratégie de classification qui se révèle largement artificielle, ce qui contribue à créer une concurrence des «bons réfugiés » politiques et des «mauvais migrants » économiques et sans papiers. Dans cette perspective, ce ne sont pas seulement les associations d'aide aux migrants qui alertent sur une essentialisation dangereuse de la problématique par le biais d'une sorte de facilité de langage; dans plusieurs articles de presse parus dans divers quotidiens français, on constate régulièrement la tendance consistant à vouloir faire le «tri » entre bons et mauvais migrants, alors que la vraie distinction est entre ceux qui ont droit à la migration, comme les réfugiés ou les familles qui se regroupent, et ceux qui ne sont acceptés que par faveur, comme les étudiants, les travailleurs, certains malades, ou ceux qui fuient la misère. De plus, le terme "migrant économique » semble être issu d'un préjugé qui discrédite les demandeurs d'asile en les renvoyant à une représentation de l'imaginaire collectif qui ne correspond pas à l'extrême complexité des réalités humaines. 

indiquées par le GDT et $\mathrm{T}$ ne renvoient qu'aux changements climatiques et environnementaux. Dans ce cas, les deux banques de données s'accordent sur le fait que « migrant » et " réfugié » sont ici synonymes, étant donné que, comme l'indique $\mathrm{T}$, l'un des points de controverse concerne le fait de savoir s'il faut qualifier les personnes déplacées par les changements du climat de "réfugiés climatiques » ou de "migrants climatiques ». Selon le droit international, l'emploi du mot « réfugié » pour désigner les personnes qui tentent d'échapper aux dérèglements de l'environnement est incorrect. La Convention des Nations Unies de 1951 et son Protocole de 1967 relatif au statut des réfugiés indiquent clairement que cette expression doit être réservée aux personnes qui fuient les persécutions.

Face à l'absence d'une définition partagée pouvant s'appuyer sur un critère juridique, le GDT et $\mathrm{T}$ considèrent comme synonymes les syntagmes suivants : "migrant/réfugié environnemental » et "migrant/réfugié climatique », les syntagmes formés par les compléments « de l'environnement » et « du climat » étant aussi acceptés («migrant/ réfugié du climat» et «migrant/réfugié de l'environnement») puisque les deux adjectifs « environnemental » et « climatique » ont ici un sens neutre, à savoir « relatif à la nature/relatif à l'environnement ». De plus, le GDT fournit d'autres informations pertinentes puisqu'il indique que le concept de «migrant climatique » est exclu de toute convention ou loi internationale et que le terme "réfugié » doit être considéré comme synonyme de " migrant » et ne doit pas être compris dans le sens que lui donne la Convention de Genève de $1951^{23}$. En ce qui concerne la question des changements climatiques, les deux ressources consultées renvoient à la dénomination « migrant 
écologique» et à la panoplie de synonymes "migrant environnemental», " écoréfugié ", "réfugié de l'environnement ", etc., bien que le GDT précise qu'il faut éviter d'employer de manière interchangeable toutes ces expressions puisque l'adjectif "écologique" ne recouvre pas en français le sens relatif uniquement à l'environnement. D'ailleurs, il en va de même pour le terme "écoréfugié » dont le préfixe « éco- » a la même valeur que l'adjectif « écologique ». Cette dernière réflexion sur l'emploi de " éco-» permet de signaler que d'autres préfixes sont à la base d'un foisonnement néologique autour des mots " migrants » et "réfugiés », tels que " pro- » et « anti-». Ceux-ci sont en effet très exploités, notamment dans la presse et dans les blogues, pour indiquer le positionnement des instances politiques face à la crise migratoire. Parmi ces néologismes obtenus par préfixation, nous signalons "promigrants ", « anti-réfugiés ", « anti-migratoire », « pro-migration », etc. ${ }^{24}$. Une dernière remarque concerne le Glossaire 2.0 du REM, dont les auteurs proposent la construction du syntagme plus complexe au niveau syntaxique «migrant poussé pour des raisons environnementales» par rapport aux syntagmes nominaux basés sur l'emploi des adjectifs « environnemental », « climatique » ou " écologique ».

Les adjectifs servant à catégoriser les migrants et les réfugiés sont également présents dans plusieurs dénominations polylexicales à deux lexèmes proposées par T. Il s'agit notamment d'adjectifs de valeur tels que " autonome ", " authentique ", « indésirable " dans des syntagmes du type « réfugié autonome » (" personne reconnue comme réfugié au sens de la Convention à l'étranger ou comme membre de la catégorie de personnes de pays d'accueil et qui dispose de ressources financières suffisantes pour subvenir à ses besoins au Canada»), "réfugié authentique ", "réfugié véritable ", "réfugié de bonne foi ", "migrant clandestin indésirable ", qui dénotent de manière évidente la posture idéologique assumée face à la condition migratoire des personnes désignées et qui servent à créer une distinction basée sur des critères prétendument juridiques.

Dans cette gradation de classification des personnes fuyant leur pays, $\mathrm{T}$ indique aussi la catégorie des "réfugiés dont personne ne veut ", à savoir ceux qui, sans être renvoyés directement dans un pays où ils seraient victimes de persécution, se voient refuser l'asile ou sont dans l'incapacité de trouver un État prêt à examiner leur cas, et vont d'un pays à l'autre en quête d'asile. T précise aussi qu'il s'agit d'une terminologie employée par le bureau Citoyenneté et Immigration Canada et par la Commission de l'immigration et du statut de réfugié.

De ce point de vue, nous partageons les réflexions de Nouss $^{25}$ sur l'émergence d'une terminologie migratoire à forte composante idéologique qui dépasse le cadre strictement juridique de classification des personnes quittant leur pays. D'après Nouss $^{26}$, dans le contexte migratoire actuel, les choix terminologiques ne sont pas innocents et le terme de "migration » semble exercer une aimantation idéologique néfaste. D'ailleurs, avec «migration légale » ou "illégale », on est encore dans une description objective, garantie par des critères juridiques qui, quoique relatifs, sont solidement ancrés et repérables. En revanche, les adjectifs «régulière » ou « irrégulière » révèlent d'emblée un changement de niveau dans la mesure où ils qualifient habituellement "immigration ", qui à la différence de "migration » induit une ordonnance spatiale et une direction («in»: du dehors vers le dedans) propices au classement hiérarchique (le dedans sera toujours mieux). De fait, les termes abandonnent une stricte neutralité puisque la «régularité» n'est pas la loi. À ce propos, nous signalons le syntagme «migrant forcé », répertorié par le Glossaire 2.0, qui 
se situe sur le même axe conceptuel que les constructions «migrant légal» et « illégal ».

Enfin, quant aux ressources officielles françaises (FT), nous constatons l'absence de fiches terminologiques sur les termes qui nous intéressent dans cette étude. Les experts du dispositif d'enrichissement de la langue française ont identifié au moment de la COP21 (Conférence des Nations Unies sur le climat en décembre 2015 à Paris) la nécessité de définir « réfugié climatique » et "réfugié environnemental ». La tâche a d'abord été confiée aux experts chargés de la terminologie et de la néologie de l'environnement, qui y ont finalement renoncé, estimant qu'il était plus problématique d'établir la distinction entre "réfugié » et "migrant " qu'entre "climatique » et « environnemental ». C'est donc les experts chargés de la terminologie et de la néologie qui ont repris l'étude des termes. Toutefois, en septembre 2018 FT ne propose toujours pas de fiche sur les termes qui nous intéressent ici. La banque de données ne présente qu'une fiche relative aux réfugiés de la mer, catégorie qui rassemble tous les termes renvoyant aux personnes qui ont fui leur pays d'origine sur une embarcation de fortune. Créée à partir de la catégorie des «boat people» anglais, la catégorie des "réfugiés de la mer » est née au cours des dernières années lors de l'interrogation relative à la manière de désigner en français les hotspot, dont les équivalents "zone d'urgence migratoire » et " point d'enregistrement » se sont imposés en français ${ }^{27}$.

La catégorisation et la typologisation des personnes ayant fui leur pays semblent donc suivre des axes et des critères fort différents. D'un côté, les motifs du déplacement sont à l'origine de plusieurs catégories très répandues et désormais acceptées dans les banques de données terminologiques et dans les dictionnaires («migrant/réfugié climatique », "migrant/réfugié environnemental»); de l'autre, les cadres juridiques motivent le recours à des syntagmes tels que "migrant/réfugié authentique", "migrant/réfugié autonome », "réfugié/migrant véritable ", qui dépassent parfois un positionnement objectif et situent la catégorisation de ces personnes au sein d'un débat idéologique sur les conditions d'acceptation et de refus dans les pays d'arrivée. Ces dénominations semblent moins stables en discours et renvoient parfois à des contextes nationaux hétérogènes. Enfin, une autre manière de catégoriser les migrants et réfugiés - plutôt neutre pour les instances politiques - est celle qui a recours à la manière de migrer ( "migrant de la mer ») et au moyen utilisé (« boat people »).

\section{Conclusion}

Les réflexions proposées ont mis en évidence qu'une réelle réflexion d'ordre sémantique existe au sein du débat public concernant l'univers de la migration. Tel que nous l'avons signalé dans la première partie de notre contribution, cette problématique investit principalement les discours médiatiques, où toute une série d'enjeux sociopolitiques et juridiques influencent de manière non négligeable les pratiques langagières et l'emploi d'une terminologie partagée relative aux flux migratoires. Dans cette perspective, il nous semble que, malgré les nombreux efforts linguistiques faits par les instances européennes, il est très difficile de suivre les rapides changements géopolitiques mondiaux provoquant de nouvelles formes de migration.

Nos recherches ont permis de mettre en évidence que la conceptualisation de la différence entre "réfugié » et "migrant » est problématique, notamment sur le plan juridique. En effet, à l'état actuel, le cadre normatif ne permet pas de catégoriser de 
façon précise et claire ces deux typologies de personnes. D'un côté, cela s'explique si l'on considère les nombreuses raisons qui sont à la base des déplacements humains qui ont récemment surgi et ont changé l'encadrement normatif indiqué dans la Convention de 1951 ; de l'autre, ce flou conceptuel serait la conséquence d'un positionnement des instances politiques qui ne veulent pas faire face à la question de la catégorisation officielle des migrants/réfugiés, ce qui provoquerait en effet un débat idéologique très profond au sein de la société contemporaine. La thématique de la migration semble vivre au gré du discours politique et du discours journalistique, où les médias fonctionnent en tant que caisse de résonance d'une terminologie qui n'est pas normalisée ni partagée par les institutions internationales.

D'un point de vue strictement linguistique, il semble que face à ce flou notionnel, de multiples dénominations nouvelles ou plus ou moins lexicalisées dans les ressources consultées sont nées, le besoin de classifier et catégoriser les personnes qui fuient leur pays étant une nécessité concrète dont les locuteurs ne peuvent pas s'affranchir. Cette donnée a été validée également dans le cadre de nos recherches sur la plateforme Néoveille, ainsi que dans les banques de données terminologiques francophones consultées, qui confirment d'ailleurs les résultats de l'enquête menée par la Coordination 75 des Sans Papiers de Paris en 2015.

L'instabilité terminologique remarquée dans cette étude est liée, comme l'indique Nouss $^{28}$, au fait que dans les nombreuses catégories de "migrants »/ » réfugiés » se mélangent, de fait, les registres ontologiques, les inscriptions historiques et les conditions sociales. D'où la nécessité, à notre avis, d'une terminologie partagée sur le plan international qui puisse représenter linguistiquement la conceptualisation complexe du phénomène migratoire contemporain.

\section{NOTES}

1. Cf. L. Calabrese, "Faut-il dire migrant ou réfugié ? Débat lexico-sémantique autour d'un problème public ", dans Langages, 210, 2018, p. 105-124 ; L. Calabrese, M. Véniard (dir.), Penser les mots, dire la migration, Paris, Academia/L'Harmattan, 2018.

2. Cf. J.-F. Sablayrolles, « De la "néologie syntaxique" à la néologie combinatoire », dans Langages, 183, 2011, p. 39-50.

3. Cf., à ce propos, les réflexions proposées par R. Raus (La terminologie multilingue. La traduction des termes de l'égalité H/F dans le discours international, Bruxelles, De Boeck, 2013) dans le cadre des grandes organisations internationales.

4. Cf. E. Cartier, « Néoveille, système de repérage et de suivi des néologismes en sept langues ", dans Neologica. Revue internationale de néologie, 10, 2016, p. 101-131.

5. Consulté au mois de septembre 2018. URL : <www.granddictionnaire.com>.

6. Consulté au mois de septembre 2018. URL : <www.culture.fr/franceterme>.

7. Consulté au mois de septembre 2018. URL : <http://www.btb.termiumplus.gc.ca/tpv2alpha/ alpha-eng.html?lang=eng>.

8. Cf. S. Moretti, T. Bonzon, «Some reflections on the IFRC's approach to migration and displacement », dans International Review of the Red Cross, 99 (1), 2017, p. 153-178. 
9. B. Vannier, «Migrants ou réfugiés : où est la frontière ?", dans Franceinfo, 29 mai 2015, consulté le 11/09/2018. URL :< http://www.francetvinfo.fr/replay-radio/le-rendez-vous-dumediateur/migrants-ou-refugies-ou-est-la-frontiere_1779309.html>.

10. Cf. K. Akoka, « Du consulat des réfugiés à l'administration des demandeurs d'asile : la fabrique des réfugiés à l'Ofpra (1952-1992) », dans e-Migrinter, 11, 2013, p. 193-197; A. Nouss, La condition de l'exilé. Penser les migrations contemporaines, Paris, Maison des Sciences de l'Homme, 2015 ; K. Akoka, «Crise des réfugiés, ou des politiques d'asile?», dans La Vie des idées, publié le 31/05/2016, consulté le 10/09/2018. URL : <http://www.laviedesidees.fr/Crise-des-refugies-ou des-politiquesd-asile.html>.

11. Cf. L. Gauvin, P. L'Hérault, A. Montandon (dir.), Le dire de l'hospitalité, Clermont-Ferrand, Presses de l'Université Blaise Pascal, 2014.

12. K. Akoka, « Du consulat des réfugiés à l'administration des demandeurs d'asile : la fabrique des réfugiés à l’ofpra (1952-1992) », cit., p. 193-197, p. 196.

13. En comparant les éditions de 2007 et de 2017 du Petit Robert (version en ligne) (s. v. «migrant» et "réfugié»), nous nous rendons compte que certaines modifications ont été intégrées sous les deux entrées. Les supports lexicographiques indiquent un phénomène incorrect de synonymie entre ces deux mots, qui est très fréquent sur le plan de l'usage, mais qui toutefois ne prend pas en considération le cadre juridique dans lequel les deux dénominations s'insèrent. D'ailleurs, le rapport d'hypéronymie-hyponymie existant entre les deux mots - tous les réfugiés sont des migrants, mais tous les migrants ne sont pas des réfugiés -, est remplacé sur le plan linguistique par un rapport de synonymie qui dans le dictionnaire n'est mentionné que sous l'entrée "migrant» («Personne qui fuit son pays pour échapper à un conflit armé », réfugié). Quant à "réfugié », alors qu'un phénomène de néologie sémantique est recensé dans Le Petit Robert («Personne ayant obtenu l'asile politique »), aucun renvoi synonymique à migrant n'est signalé.

14. C. Rodier, Migrants \& Réfugiés. Réponse aux indécis, aux inquiets et aux réticents, Paris, La Découverte, 2016, p. 15.

15. Cf. R. Setti, «Migranti, profughi e rifugiati. Anche le parole delle migrazioni sono sempre in viaggio", Firenze, Accademia della Crusca, 2017. Consulté le 25/09/2018. URL: <http:// www.accademiadellacrusca.it/it/lingua-italiana/consulenza-linguistica/domande-risposte/ migranti-profughi-rifugiati-anche-parole-mig>.

16. Nous signalons ici le même phénomène sémantique indiqué par Vadot pour les mots « insertion » et « intégration ». Vadot remarque que «insertion et intégration semblent désormais souvent nommer la même réalité, voire être interchangeables. Pour autant, l'exploration du sentiment linguistique de certains locuteurs permet de faire apparaître le poids de mémoires discursives différenciées se rapportant à chacune des dénominations mises en contraste " ( $M$. Vadot, « De quoi Intégration est-il le nom ? L'importation d'une querelle de mots dans le champ de la formation linguistique des migrants ", dans Argumentation et Analyse du Discours, 17, 2016, publié le 15 octobre 2016. Consulté le 07/10/2018. URL : <https://journals.openedition.org/aad/ 2228>).

17. Les résultats de l'enquête menée sont disponibles à l'adresse suivante: <https:// csp75.wordpress.com/qui-dit-quoi/>.

18. La parution de ces textes remonte à la période novembre 2014-novembre 2015. Sauf pour les médias, les textes de positionnement, communiqués, appels à action et argumentaires ont été privilégiés.

19. Cf. G. Parrinello, F. Gemenne, "Qu'est-ce qu'un réfugié environnemental ? ", dans Colloque annuel de Blois Les rendez-vous de l'histoire, 6-8 octobre 2016, à paraître.

20. Cf. Ibidem ; C. Rodier, op. cit., p. 14-15.

21. Pour ce qui est des ressources lexicographiques concernant la migration, nous signalons, d'une part, le glossaire (URL : <http://publications.iom.int/system/files/pdf/iml_9_fr.pdf et ci- 
joint>) et son résumé Termes clés de la migration (URL : <https://www.iom.int/fr/termes-cles-dela-migration>) de l'Organisation internationale pour les migrations (OIM), qui dépend de l'ONU, et, d'autre part, la banque UNTERM (banque de données des Nations Unies, URL <https:// unterm.un.org/UNTERM/search>). À ce propos, nous précisons que les banques de données terminologiques en ligne que nous avons consultées ne font pas (ou quasiment pas) référence à la terminologie OIM/ONU, qui est quand même une terminologie de référence en français, le français étant une des langues officielles de l'ONU et de l'OIM.

22. Nous avons sélectionné ces banques de données terminologiques en raison du fait qu'elles sont développées dans des contextes de travail francophones. Les données retenues pourront être intégrées avec celles proposées dans d'autres banques de données terminologiques conçues dans une optique de travail plurilingue, telles que, à titre d'exemple, IATE.

23. $T$ semble s'accorder avec les informations fournies par le GDT puisqu'il indique que «l'un des points de controverse qui surgit aussitôt est celui de savoir s'il faut qualifier les personnes déplacées par les changements du climat de 'réfugiés climatiques' ou de 'migrants climatiques'. Au regard du droit international, le mot 'réfugié' appliqué aux personnes qui tentent d'échapper aux dérèglements de l'environnement, n'est pas tout à fait correct. La Convention des Nations Unies de 1951 et son Protocole de 1967 relatif au statut des réfugiés indiquent clairement que cette expression doit être réservée aux personnes qui fuient les persécutions [...] ».

24. Cf. C. Grimaldi, » Identité et diversité dans la terminologie de la crise migratoire : entre crise sociale et crise langagière ", dans C. Diglio, C. Napolitano, F. Perilli (dir.), Identité, Diversité et Langue, entre ponts et murs. Hommage à Giulia Papoff, Napoli, Loffredo Editore, 2018, p. 163-174.

25. Cf. A. Nouss, op. cit.

26. A. Nouss, op. cit., p. 14.

27. Nous remercions Étienne Quillot de la Délégation générale à la langue française et aux langues de France (DGLFLF) pour les documents qu'il nous a fournis et pour ses précieuses suggestions sur les travaux menés par la DGLFLF.

28. A. Nouss, op. cit., p. 21-22.

\section{RÉSUMÉS}

Le débat politique sur la crise migratoire pose de nombreux problèmes quant à la catégorisation des migrants et réfugiés, dont la définition juridique ne satisfait pas les nécessités de désignation. Dans cette contribution, nous nous proposons d'explorer la terminologie de la crise migratoire à l'aide de plateformes et banques de données terminologiques. Notre but est de fournir une réflexion sur ce qui motive les locuteurs et les organes de presse à opter pour l'un ou l'autre de ces termes.

The political debate on the migration crisis poses many problems with reference to the categorization of migrants and refugees, whose legal definition does not satisfy the designation needs. This contribution aims to explore the terminology of migration through online terminology platforms and databases. The contribution provides a framework of the choices that lead to use one term over another in the context of migration terminology. 
INDEX

Keywords : migration crisis, terminology, denomination, corpus, databases

Mots-clés : crise migratoire, terminologie, dénomination, corpus, banques de données 